\title{
Technique for the experimental estimation of nonlinear energy transfer in fully developed turbulence
}

\author{
J. S. Kim, R. D. Durst, and R. J. Fonck \\ Department of Nuclear Engineering and Engineering Physics, University of Wisconsin, \\ Madison, Wisconsin 53706 \\ E. Fernandez, A. Ware, and P. W. Terry \\ Department of Physics, University of Wisconsin, Madison, Wisconsin 53706
}

(Received 3 June 1996; accepted 12 August 1996)

\begin{abstract}
A new procedure for calculating the nonlinear energy transfer and linear growth/damping rate of fully developed turbulence is derived. It avoids the unphysically large damping rates typically obtained using the predecessor method of Ritz [Ch. P. Ritz, E. J. Powers, and R. D. Bengtson, Phys. Fluids B 1, 153 (1989)]. It enforces stationarity of the turbulence to reduce the effects of noise and fluctuations not described by the basic governing equation, and includes the fourth-order moment to avoid the closure approximation. The new procedure has been implemented and tested on simulated, fully developed two-dimensional (2-D) turbulence data from a 2-D trapped-particle fluid code, and has been shown to give excellent reconstructions of the input growth rate and nonlinear coupling coefficients with good noise rejection. However, in the experimentally important case where only a one-dimensional (1-D) averaged representation of the underlying 2-D turbulence is available, this technique does not, in general, give acceptable results. A new 1-D algorithm has thus been developed for analysis of 1-D measurements of intrinsically 2-D turbulence. This new 1-D algorithm includes the nonresonant wave numbers in calculating the bispectra, and generally gives useful results when the width of the radial wave number spectrum is comparable to or less than that of the poloidal spectrum. (c) 1996 American Institute of Physics. [S1070-664X(96)03811-6]
\end{abstract}

\section{INTRODUCTION}

The characterization and identification of the type of mode associated with the anomalous transport of heat and particles in high-temperature tokamak plasmas are topics of considerable interest since they might provide insight into ways of reducing the anomalous losses. Recently, bispectral analysis has emerged as a way to experimentally estimate the linear growth rate and nonlinear energy $\operatorname{transfer}^{1-8}$ in fully developed plasma turbulence, and thereby provide a more direct tool for mode identification compared to early transport experiments, which relied on indirect comparisons to theoretical expectations. ${ }^{3}$

Bispectral analysis, which measures the amount of phase correlation between three spectral components, is used to investigate nonlinear wave-wave interactions. Although there have been many experimental studies that used digital bispectral analysis in fluids and plasmas to demonstrate that nonlinear energy transfer by three-wave coupling processes is important, ${ }^{4-8}$ the use of this analysis technique was typically limited to qualitative applications. In the late 1980's Ritz et al. developed an analysis method that uses experimental turbulence data and enables one to make a quantitative estimation of the nonlinear coupling coefficients and the resulting amount of energy cascading between waves in a fully developed turbulent system. ${ }^{1-3}$ However, application of Ritz's method suffered from the appearance of nonphysical behavior of the derived linear growth rate due to nonideal power, here defined as noise, systematic errors, higher-order coupling, wave-particle coupling, etc.

In this work, a new procedure based on Ritz's approach ${ }^{3}$ for calculating the nonlinear energy transfer and linear growth rate of fully developed turbulence is presented. It enforces a stationarity condition to eliminate nonideal power and utilizes the fourth-order moment to avoid errors arising from the closure approximation. The validity of the procedure is examined numerically with data generated from a turbulence model. This computational experiment estimates how well the procedure can extract relevant physical results from raw fluctuation data and tests the validity of its application to analysis of one-dimensional (1-D) experimental measurements of intrinsically two-dimensional (2-D) turbulence.

The remainder of this paper is organized as follows. In Sec. II, the nonlinear drift wave coupling equation is presented as the governing equation. In Sec. III we describe how to make a quantitative estimation of the linear growth rate and of the amount of energy cascading between waves for a fully turbulent system. Furthermore, the problems associated with the Ritz method are identified and the modified Ritz method that overcomes these problems is developed and discussed. In Sec. IV, we apply these procedures to pure 1-D and 2-D simulated turbulence data, and discuss how well the procedure reconstructs the input variables of the simulation data. In Sec. V, we discuss the practically important case where only 1-D radially averaged data is available rather than a complete 2-D characterization of the plasma turbulence. In this case, it is found that, in general, it is not possible to completely reconstruct the linear growth rate and nonlinear coupling coefficients. However, techniques are discussed that can derive a useful, partial characterization of the fluctuations. The summary and conclusion of this work are presented in Sec. VI. 


\section{ANALYSIS MODEL}

In the late 1980s, new fluctuation diagnostics such as beam emission spectroscopy ${ }^{9,10}$ (BES), heavy ion beam probe $^{11}$ (HIBP), and correlation reflectrometry ${ }^{12}$ (CR) showed that core density fluctuations have a broad wave number spectrum that is strongly peaked at long wavelengths, $\left\langle k_{\theta}\right\rangle \rho_{s} \leqslant 0.1{ }^{13}$ The broad spectrum appears to indicate a strongly turbulent state, in that the interaction between the separate waves broadens out the frequency spectrum, which means a linear analysis is unable to fully characterize the turbulent spectrum. Recently, many experiments have used bispectral analysis to demonstrate that three-wave coupling processes are important in fluids ${ }^{14,15}$ and plasmas. ${ }^{1-8}$ This three-wave coupling term can be theoretically derived from the convective derivative [i.e., $(\vec{v} \cdot \nabla) \vec{v}$, where $\vec{v}$ is the flow velocity] in neutral fluids or the polarization drift and $\widetilde{E} \times B$ drift convection in plasmas. ${ }^{3}$

To make quantitative estimates of the linear growth rate and the energy cascade rate between waves for fully developed turbulence, we consider the nonlinear drift wave coupling equation written in the form ${ }^{3}$

$$
\begin{aligned}
\frac{\partial \varphi(k, t)}{\partial t}= & \Lambda_{k}^{L} \varphi(k, t)+\frac{1}{2} \sum_{\substack{k_{1}, k_{2} \\
k=k_{1}+k_{2}}} \Lambda_{k}^{Q}\left(k_{1}, k_{2}\right) \\
& \times \varphi\left(k_{1}, t\right) \varphi\left(k_{2}, t\right) \\
= & \Lambda_{k}^{L} \varphi(k, t)+\sum_{\substack{k \geqslant k_{2} \\
k=k_{1}+k_{2}}} \Lambda_{k}^{Q}\left(k_{1}, k_{2}\right) \\
& \times \varphi\left(k_{1}, t\right) \varphi\left(k_{2}, t\right),
\end{aligned}
$$

where $\varphi(k, t)$ is the density fluctuation at wave number $k$ defined by $\varphi(x, t)=\Sigma_{k} \varphi(k, t) e^{i k x}, \Lambda_{k}^{L}$ is the linear transfer function, and $\Lambda_{k}^{O}$ is the nonlinear transfer function. This single-field drift wave model, which assumes a linear phase relation between fields (such as density, potential, ion temperature, etc.), reproduces many, but not all, features of drift wave turbulence.

The governing equation (1), which is appropriate to describe turbulence that is dominated by three-wave coupling, shows that the rate of change of the spatial Fourier spectrum of the fluctuating field is determined only by linear and quadratic nonlinear interactions. The linear transfer function $\Lambda_{k}^{L}$ encompasses linear damping and growth, spectral broadening (linear dispersion), the diamagnetic driving term, etc. The nonlinear transfer function $\Lambda_{k}^{Q}$ gives the strength of mode coupling due to the convective derivative terms such as the nonlinear polarization drift and $\widetilde{E} \times B$ advection of density in the drift wave model.

To get the energy cascade and linear growth rate of modes at given $k$, the time derivative in Eq. (1) is replaced by a finite difference and ensemble averaged over many statistically similar realizations to make Eq. (1) statistically meaningful.

If the spectrum $\varphi(k, t)$ is characterized by a complex representation (i.e., amplitude and phase $[\varphi(k, t)$ $\left.\left.=|\varphi(k, t)| e^{i \Theta(k, t)}\right]\right)$, the change of the spectrum in time can be estimated by a finite difference approach:

$$
\begin{aligned}
\frac{\partial \varphi(k, t)}{\partial t}= & \lim _{\tau \rightarrow 0}\left(\frac{|\varphi(k, t+\tau)|-|\varphi(k, t)|}{\tau|\varphi(k, t)|}\right. \\
& \left.+i \frac{\Theta(k, t+\tau)-\Theta(k, t)}{\tau}\right) \varphi(k, t) .
\end{aligned}
$$

Substituting Eq. (2) into Eq. (1) and simplifying, we obtain

$$
Y_{k}=L_{k} X_{k}+\sum_{\substack{k_{1} \geqslant k_{2} \\ k=k_{1}+k_{2}}} Q_{k}^{k_{1}, k_{2}} X_{k_{1}} X_{k_{2}},
$$

where

$$
\begin{aligned}
& X_{k}=\varphi(k, t), \quad Y_{k}=\varphi(k, t+\tau), \\
& L_{k}=\frac{\Lambda_{k}^{L} \tau+1-i[\Theta(k, t+\tau)-\Theta(k, t)]}{e^{-i[\Theta(k, t+\tau)-\Theta(k, t)]},} \\
& Q_{k}^{k_{1}, k_{2}}=\frac{\Lambda_{k}^{Q}\left(k_{1}, k_{2}\right) \tau}{e^{-i[\Theta(k, t+\tau)-\Theta(k, t)]} .}
\end{aligned}
$$

Equation (3) models an arbitrary nonlinear system with a parallel combination of linear $\left(L_{k}\right)$ and quadratic transfer functions $\left(Q_{k}^{k_{1}, k_{2}}\right)$.

To obtain the general form of the linear growth rate $\left(\gamma_{k}\right)$ and the rate of nonlinear energy transfer $\left(T_{k}\right)$, we introduce the energy flow equation as a difference equation given by

$$
\frac{\partial P_{k}}{\partial t} \approx \frac{\left\langle Y_{k} Y_{k}^{*}\right\rangle-\left\langle X_{k} X_{k}^{*}\right\rangle}{\tau}=\gamma_{k} P_{k}+T_{k},
$$

where $P_{k}=\left\langle X_{k} X_{k}^{*}\right\rangle$. Equation (4), which describes the time rate of change of the spectral power of a wave number $k$, gives the capability of experimentally measuring the linear growth/damping rate and the rate of nonlinear energy transfer in fully developed turbulence. The nonlinear energy transfer rate, $T_{k}$, represents a net flow of energy into or away from a given mode $k$. That is, positive $T_{k}$ represents a net flow of energy into the mode $k$; negative $T_{k}$ represents net outflow. By multiplying Eq. (3) with the complex conjugate of $Y_{k}$ and ensemble averaging over many statistically similar realizations (denoted by angular brackets), we obtain

$$
\begin{aligned}
\left\langle Y_{k} Y_{k}^{*}\right\rangle= & L_{k}\left\langle X_{k} Y_{k}^{*}\right\rangle+\sum_{\substack{k_{1} \geqslant k_{2} \\
k=k_{1}+k_{2}}} Q_{k}^{k_{1}, k_{2}}\left\langle X_{k_{1}} X_{k_{2}} Y_{k}^{*}\right\rangle \\
= & L_{k}\left\langle X_{k}\left[L_{k}^{*} X_{k}^{*}+\sum_{\substack{k_{1} \geqslant k_{2} \\
k=k_{1}+k_{2}}} Q_{k}^{k_{1}, k_{2}^{*}} X_{k_{1}}^{*} X_{k_{2}}^{*}\right]\right\rangle \\
& +\left\langle\sum_{\substack{k_{1} \geqslant k_{2} \\
k=k_{1}+k_{2}}} Q_{k}^{k_{1}, k_{2}} X_{k_{1}}, X_{k_{2}}\left(L_{k}^{*} X_{k}^{*}\right.\right. \\
& \left.\left.+\sum_{\substack{k_{1}^{\prime} \geqslant k_{2}^{\prime} \\
k=k_{1}^{\prime}+k_{2}^{\prime}}} Q_{k}^{k_{1}^{\prime}, k_{2}^{\prime *}} X_{k_{1}^{\prime}}^{*} X_{k_{2}^{\prime}}^{*}\right)\right\rangle .
\end{aligned}
$$


Substituting Eq. (5) into Eq. (4) and simplifying, we finally obtain

$$
\begin{aligned}
& \gamma_{k} \approx \frac{\left|L_{k}\right|^{2}-1}{\tau}, \\
& T_{k} \approx 2 \operatorname{Re}\left(L_{k}^{*} \sum_{\substack{k_{1} \geqslant k_{2} \\
k=k_{1}+k_{2}}} \frac{Q_{k}^{k_{1}, k_{2}}\left\langle X_{k_{1}} X_{k_{2}} X_{k}^{*}\right\rangle}{\tau}\right) \\
&+\sum_{\substack{k_{1}^{\prime} \geqslant k_{2}^{\prime} \\
k=k_{1}^{\prime}+k_{2}^{\prime}}} \sum_{\substack{k_{1} \geqslant k_{1}+k_{2} \\
k=k_{1}+k_{2}}} \frac{Q_{k}^{k_{1}, k_{2}} Q_{k}^{k_{1}^{\prime}, k_{2}^{\prime *}}\left\langle X_{k_{1}} X_{k_{2}} X_{k_{1}^{\prime}}^{*} X_{k_{2}^{\prime}}^{*}\right\rangle}{\tau} .
\end{aligned}
$$

In the next section, we will derive moment equations to obtain the unknown linear $\left(L_{k}\right)$ and quadratic transfer functions $\left(Q_{k}^{k_{1}, k_{2}}\right)$ by manipulating Eq. (3). These unknown linear $\left(L_{k}\right)$ and quadratic $\left(Q_{k}^{k_{1}, k_{2}}\right)$ transfer functions determine the linear growth rate and the nonlinear energy transfer rate from the above equation.

\section{PROCEDURE TO ESTIMATE POWER TRANSFER}

\section{A. Review of Ritz method}

Moment equations were derived by Ritz et al. ${ }^{1-3}$ to estimate the linear and quadratic transfer functions for nonGaussian input signals. By multiplying Eq. (3) with the complex conjugate of $X_{k}$ and ensemble averaging over many statistically similar realizations, we obtain the second moment equation:

$$
\left\langle Y_{k} X_{k}^{*}\right\rangle=L_{k}\left\langle X_{k} X_{k}^{*}\right\rangle+\sum_{\substack{k_{1} \geqslant k_{2} \\ k=k_{1}+k_{2}}} Q_{k}^{k_{1}, k_{2}}\left\langle X_{k_{1}} X_{k_{2}} X_{k}^{*}\right\rangle .
$$

By multiplying Eq. (3) with $X_{k_{1}^{\prime}}^{*} X_{k_{2}^{\prime}}^{*}$ and ensemble averaging, we obtain a third equation containing the fourth-order moment $\left\langle X_{k_{1}} X_{k_{2}} X_{k_{1}^{\prime}}^{*} X_{k_{2}^{\prime}}^{*}\right\rangle$. To close the expansion of the higher moments, the fourth-order moment is approximated by the square of the second-order moments $\left\langle\left|X_{k_{1}} X_{k_{2}}\right|^{2}\right\rangle$ by neglecting terms with $\left(k_{1}^{\prime}, k_{2}^{\prime}\right) \neq\left(k_{1}, k_{2}\right)$, as was proposed by Millionshchikov. ${ }^{16}$ The simplified third moment equation is then

$$
\left\langle Y_{k} X_{k_{1}}^{*} X_{k_{2}}^{*}\right\rangle=L_{k}\left\langle X_{k} X_{k_{1}}^{*} X_{k_{2}}^{*}\right\rangle+Q_{k}^{k_{1}, k_{2}}\left\langle\left|X_{k_{1}} X_{k_{2}}\right|^{2}\right\rangle .
$$

Ritz et al. used Eqs. (6) and (7), which we will refer to as the Ritz method, to permit the quantitative estimation of the transfer functions for general input signals. The derived linear transfer function is

$$
L_{k}=\frac{\left\langle X_{k}^{*} Y_{k}\right\rangle-\sum_{\substack{k_{1} \geqslant k_{2} \\ k=k_{1}+k_{2}}} \frac{\left\langle X_{k}^{*} X_{k_{1}} X_{k_{2}}\right\rangle\left\langle Y_{k} X_{k_{1}}^{*} X_{k_{2}}^{*}\right\rangle}{\left\langle\left|X_{k_{1}} X_{k_{2}}\right|^{2}\right\rangle}}{\left\langle X_{k} X_{k}^{*}\right\rangle-\sum_{\substack{k_{1} \geqslant k_{2} \\ k=k_{1}+k_{2}}} \frac{\left|\left\langle X_{k}^{*} X_{k_{1}} X_{k_{2}}\right\rangle\right|^{2}}{\left\langle\left|X_{k_{1}} X_{k_{2}}\right|^{2}\right\rangle}} .
$$

However, application of this method can often yield unphysically large damping coefficients at all spectral components for the measured fluctuation data, as noted by Ritz. ${ }^{1}$ These unrealistic results arise because the method does not account for nonideal fluctuations [i.e., fluctuations not described by Eq. (1) such as noise in the measured signal, higher-order nonlinear coupling, systematic errors, etc.]. That is, the autopower spectrum in the denominator of Eq. (8) includes nonideal fluctuations because phase relations between wave number components are suppressed in the auto power spectrum. This leads to the unphysically large damping obtained in the Ritz method. To rectify this, we reconstruct the auto power spectrum (contaminated by nonideal power) by using higher-order moments that preserve the Fourier phase of a signal.

\section{B. Modified Ritz method}

To correct for nonideal effects, we enforce a temporal stationarity condition that allows us to extract that portion of the measured power for which Eq. (1) provides an adequate description. In essence, this involves the removal of any nonideal fluctuation power inherent in the measured spectrum but uncorrelated with the fluctuation power described by the physics contained in Eq. (1). The degree of relevance of this overall analysis then depends on how much of the ideal fluctuation power remains in the wave-wave coupling model spectrum compared to the total turbulence power. If a large fraction of the turbulence power is well described by the linear and quadratic wave-wave coupling model in Eq. (1), this analysis can be expected to shed light on the dynamics of the nonlinear mode structure of the turbulence. However, it can be considered irrelevant if almost all the turbulent power is eliminated by this approach.

In this work, we describe the measured spectra $\left(X_{k}, Y_{k}\right)$ to be the sum of an ideal spectrum $\left(\beta_{k}, \alpha_{k}\right)$, which is driven by linear and quadratic processes described by Eq. (1) and a nonideal spectrum $\left(X_{k}^{n i}, Y_{k}^{n i}\right)$ that does not participate in linear and three-wave coupling processes. That is,

$$
X_{k}=\beta_{k}+X_{k}^{n i}, \quad Y_{k}=\alpha_{k}+Y_{k}^{n i} .
$$

Using Eq. (9) we can rewrite Eq. (3) in the form

$$
\begin{aligned}
\left(Y_{k}-Y_{k}^{n i}\right)= & L_{k}\left(X_{k}-X_{k}^{n i}\right)+\sum_{\substack{k_{1} \geqslant k_{2} \\
k=k_{1}+k_{2}}} Q_{k}^{k_{1}, k_{2}}\left(X_{k_{1}}-X_{k_{1}}^{n i}\right) \\
& \times\left(X_{k_{2}}-X_{k_{2}}^{n i}\right)
\end{aligned}
$$

where the finite difference for the ideal spectrum is expressed only in terms of the measured and nonideal spectra. We assume here that the nonideal spectrum is completely uncorrelated with the ideal fluctuation spectrum $\left(\beta_{k}, \alpha_{k}\right)$, as is appropriate for noise or any spectrum not described by the physics of Eq. (1).

By multiplying Eq. (10) with the complex conjugate of $X_{k}, Y_{k}$, and $X_{k_{1}^{\prime}} X_{k_{2}^{\prime}}$, ensemble averaging over many statistically similar realizations (as used in Ritz method), and re- 
moving all cross terms containing the nonideal spectrum except the autopower spectra $\left(\left\langle X_{k}^{n i} X_{k}^{n i^{*}}\right\rangle,\left\langle Y_{k}^{n i} Y_{k}^{n i *}\right\rangle\right)$, we obtain three moment equations:

$$
\begin{gathered}
\left\langle Y_{k} X_{k}^{*}\right\rangle=L_{k}\left\langle\beta_{k} \beta_{k}^{*}\right\rangle+\sum_{\substack{k_{1} \geqslant k_{2} \\
k=k_{1}+k_{2}}} Q_{k}^{k_{1}, k_{2}}\left\langle X_{k_{1}} X_{k_{2}} X_{k}^{*}\right\rangle, \\
\left\langle\alpha_{k} \alpha_{k}^{*}\right\rangle=L_{k}\left\langle X_{k} Y_{k}^{*}\right\rangle+\sum_{\substack{k_{1} \geqslant k_{2} \\
k=k_{1}+k_{2}}} Q_{k}^{k_{1}, k_{2}}\left\langle X_{k_{1}} X_{k_{2}} Y_{k}^{*}\right\rangle, \\
\left\langle Y_{k} X_{k_{1}}^{*} X_{k_{2}}^{*}\right\rangle=L_{k}\left\langle X_{k} X_{k_{1}}^{*} X_{k_{2}}^{*}\right\rangle \\
+\sum_{\substack{k_{1}^{\prime} \geqslant k_{2}^{\prime} \\
k=k_{1}^{\prime}+k_{2}^{\prime}=k_{1}+k_{2}}} Q_{k}^{k_{1}^{\prime}, k_{2}^{\prime}}\left\langle X_{k_{1}^{\prime}} X_{k_{2}^{\prime}} X_{k_{1}}^{*} X_{k_{2}}^{*}\right\rangle .
\end{gathered}
$$

In this case, we will retain the full fourth-order cumulant $\left\langle X_{k_{1}^{\prime}} X_{k_{2}^{\prime}} X_{k_{1}}^{*} X_{k_{2}}^{*}\right\rangle$ rather than using a closure approximation, as was done previously. In most physical systems, the random phase assumption is valid and the second-order closure approximation is valid. However, in some numerical experiments the random phase assumption is violated and in this case one must use the exact fourth-order cumulant, despite its greater computational cost.
One more relationship is needed to complete the set for the derivation of the four unknown variables $L_{k}, Q_{k}^{k_{1}, k_{2}}$, $\left\langle\beta_{k} \beta_{k}^{*}\right\rangle$, and $\left\langle\alpha_{k} \alpha_{k}^{*}\right\rangle$. For fully developed turbulence [stationary turbulence, $\left.\partial P_{k} / \partial t \approx\left(\left\langle\alpha_{k} \alpha_{k}^{*}\right\rangle-\left\langle\beta_{k} \beta_{k}^{*}\right\rangle\right) / \tau=0\right]$, it is clear that the linear growth of instabilities is balanced by the nonlinear energy transfer. This means that the power spectrum is forced to be fully saturated by only the linear and three mode coupling terms in the model of our system. This observation suggests the fourth equation to be in the form of

$$
\left\langle\alpha_{k} \alpha_{k}^{*}\right\rangle=\left\langle\beta_{k} \beta_{k}^{*}\right\rangle .
$$

This stationarity condition is equivalent to an assumption of local homogeneity when we apply the procedure to 1-D experimental turbulence data. Expressed in matrix notation, the complete set of equations is then

$$
\begin{aligned}
& \left\langle Y_{k} X_{k}^{*}\right\rangle=L_{k}\left\langle\beta_{k} \beta_{k}^{*}\right\rangle+\mathbf{Q} \cdot \mathbf{A}, \\
& \left\langle\alpha_{k} \alpha_{k}^{*}\right\rangle=L_{k}\left\langle X_{k} Y_{k}^{*}\right\rangle+\mathbf{Q} \cdot \mathbf{B}, \\
& \left(\mathbf{B}^{*}\right)^{T}=L_{k}\left(\mathbf{A}^{*}\right)^{T}+\mathbf{Q} \cdot \mathbf{F}, \\
& \left\langle\alpha_{k} \alpha_{k}^{*}\right\rangle=\left\langle\beta_{k} \beta_{k}^{*}\right\rangle,
\end{aligned}
$$

where in the case in which the index $l$ of the wave number $k$ is an even number,

$$
\begin{aligned}
& \mathbf{Q}=\left(Q_{l}^{l / 2, l / 2}, Q_{l}^{(l+2) / 2,(l-2) / 2}, Q_{l}^{(l+4) / 2,(l-4) / 2}, \ldots, Q_{l}^{l_{N}, l-l_{N}}\right), \\
& \mathbf{A}=\left(\left\langle X_{l / 2} X_{l / 2} X_{l}^{*}\right\rangle,\left\langle X_{(l+2) / 2} X_{(l-2) / 2} X_{l}^{*}\right\rangle,\left\langle X_{(l+4) / 2} X_{(l-4) / 2} X_{l}^{*}\right\rangle, \ldots,\left\langle X_{l_{N}} X_{l-l_{N}} X_{l}^{*}\right\rangle\right)^{T}, \\
& \mathbf{B}=\left(\left\langle X_{l / 2} X_{l / 2} Y_{l}^{*}\right\rangle,\left\langle X_{(l+2) / 2} X_{(l-2) / 2} Y_{l}^{*}\right\rangle,\left\langle X_{(l+4) / 2} X_{(l-4) / 2} Y_{l}^{*}\right\rangle, \ldots,\left\langle X_{l_{N}} X_{l-l_{N}} Y_{l}^{*}\right\rangle\right)^{T}, \\
& \mathrm{~F}=\left(\begin{array}{cclc}
\left\langle X_{l / 2} X_{l / 2} X_{l / 2}^{*} X_{l / 2}^{*}\right\rangle & \left\langle X_{l / 2} X_{l / 2} X_{(l+2) / 2}^{*} X_{(l-2) / 2}^{*}\right\rangle & \cdot & \cdot \\
\left\langle X_{(l+2) / 2} X_{(l-2) / 2} X_{l / 2}^{*} X_{l / 2}^{*}\right\rangle & \left\langle X_{(l+2) / 2} X_{(l-2) / 2} X_{(l+2) / 2}^{*} X_{(l-2) / 2}^{*}\right\rangle & \cdot & \cdot \\
\cdot & \cdot & \cdot & \cdot \\
\cdot & \cdot & \cdot & \left\langle X_{l_{N}} X_{l-l_{N}} X_{l_{N}}^{*} X_{l-l_{N}}^{*}\right\rangle
\end{array}\right) .
\end{aligned}
$$

Note that $l$ is used here for the index of the mode wave number $k(k=f(l)$, where $l$ is an integer and $f$ is a linear function), and similar definitions are also used for the odd index of $k$.

Here the diagonal terms of the fourth-order moment $(\mathbf{F})$ are the square of the second-order moment that the Ritz method employs in order to make a closure. Thus, the use of the fourth-order moment in this modified Ritz method mathematically complements the Ritz method but allows a description of the governing equation (1) without the closure approximation.

This set of equations is directly solved to obtain the four unknown variables. Rearranging Eq. (13), we have

$$
\mathbf{Q}=\left(\mathbf{B}^{*}\right)^{T} \cdot \mathrm{F}^{-1}-L_{k}\left(\mathbf{A}^{*}\right)^{T} \cdot \mathbf{F}^{-1} .
$$

Substituting Eq. (15) into Eqs. (11) and (12), we have two expressions for $L_{k}$ in the form of

$$
L_{k}=\frac{\left\langle Y_{k} X_{k}^{*}\right\rangle-\left(\mathbf{B}^{*}\right)^{T} \cdot \mathrm{F}^{-1} \cdot \mathbf{A}}{\left\langle\beta_{k} \beta_{k}^{*}\right\rangle-\left(\mathbf{A}^{*}\right)^{T} \cdot \mathbf{F}^{-1} \cdot \mathbf{A}},
$$

and

$$
L_{k}=\frac{\left\langle\alpha_{k} \alpha_{k}^{*}\right\rangle-\left(\mathbf{B}^{*}\right)^{T} \cdot \mathbf{F}^{-1} \cdot \mathbf{B}}{\left\langle X_{k} Y_{k}^{*}\right\rangle-\left(\mathbf{A}^{*}\right)^{T} \cdot \mathbf{F}^{-1} \cdot \mathbf{B}} .
$$

Using Eqs. (16) and (17), we can then solve for the linear growth rate given by

$$
\gamma_{k}=\frac{1}{\tau} \frac{\left(\mathbf{A}^{*}\right)^{T} \cdot \mathbf{F}^{-1} \cdot \mathbf{A}-\left(\mathbf{B}^{*}\right)^{T} \cdot \mathbf{F}^{-1} \cdot \mathbf{B}}{\left\langle\beta_{k} \beta_{k}^{*}\right\rangle-\left(\mathbf{A}^{*}\right)^{T} \cdot \mathbf{F}^{-1} \cdot \mathbf{A}} .
$$

In Eq. (18), the linear portion $\left(\left\langle\beta_{k} \beta_{k}^{*}\right\rangle-\left(\mathbf{A}^{*}\right)^{T}\right.$ $\left.\cdot \mathrm{F}^{-1} \cdot \mathbf{A}\right)$ of total power $\left(\left\langle\beta_{k} \beta_{k}^{*}\right\rangle\right)$ at wave number $k$ is growing (i.e., outflow of linear energy) in order to saturate the total fluctuation power if the flow of nonlinear energy 
into wave number $k\left[T_{\text {in }}=\left(\mathbf{A}^{*}\right)^{T} \cdot \mathbf{F}^{-1} \cdot \mathbf{A}\right]$ is bigger than the outflow of nonlinear energy $\left[T_{\text {out }}=\left(\mathbf{B}^{*}\right)^{T} \cdot \mathrm{F}^{-1} \cdot \mathbf{B}\right]$. This suggests that the amplitudes of the auto-bispectrum and crossbispectrum are very important in this procedure in determining which wave numbers linearly grow or damp.

The numerical estimations of well-known statistical quantities such as the autopower spectrum $P_{k}=\left\langle X_{k} X_{k}^{*}\right\rangle$, the cross-power spectrum $\left\langle Y_{k} X_{k}^{*}\right\rangle$, the auto-bispectrum $\left\langle X_{k_{1}+k_{2}} X_{k_{1}}^{*} X_{k_{2}}^{*}\right\rangle$, and the cross-bispectrum $\left\langle Y_{k_{1}+k_{2}} X_{k_{1}}^{*} X_{k_{2}}^{*}\right\rangle$, are thoroughly discussed in Refs. 2, 3, 17, 18, and 19 and are not discussed here.

To summarize, this procedure postulates that the measured spectrum consists of two components:

$$
X_{k}=\beta_{k}+X_{k}^{n i},
$$

where $\beta_{k}$ is the spectrum due to the processes described by Eq. (1) (linear growth/damping and three-wave coupling) and $X_{k}^{n i}$ is the spectrum due to processes not described by Eq. (1) such as higher-order nonlinear coupling, systematic errors, and noise. By enforcing the stationarity condition $\left(\left\langle\alpha_{k} \alpha_{k}^{*}\right\rangle=\left\langle\beta_{k} \beta_{k}^{*}\right\rangle\right)$ we are able to solve for that portion of the spectrum that can be described by linear and quadratic (three-wave coupling) terms.

However, note that no detailed information about those processes that drive the extra fluctuation power is obtained by this procedure. As mentioned earlier, the procedure must be justified after the fact by comparing the linear-quadratic power, $\left\langle\beta_{k} \beta_{k}^{*}\right\rangle$, to the nonideal power, $\left\langle X_{k}^{n i} X_{k}^{n i *}\right\rangle$. For the case where $\left\langle\beta_{k} \beta_{k}^{*}\right\rangle \gg\left\langle X_{k}^{n i} X_{k}^{n i *}\right\rangle$ we conclude that the plasma fluctuations are indeed dominated by linear and quadratic processes and the nonlinear energy transfer and growth rate estimates obtained are meaningful.

\section{SIMULATION EXPERIMENTS}

To check how well the derived nonlinear characteristics compare to the input values, we apply the bispectral analysis to fully developed, simulated turbulence data. This numerical experiment demonstrates the capability of the procedure to reconstruct the input linear growth rate and nonlinear coupling coefficients. The influence of noise will also be investigated with random Gaussian noise.

\section{A. Test of the procedure with pure 1-D turbulence data}

The first test of the Ritz method and the modified Ritz method uses a 1-D model. To generate saturated 1-D nonlinear data, we analytically define the linear and quadratic transfer functions and numerically integrate the basic Eq. (1), starting from Gaussian random spectra, to obtain fully developed turbulence. This simulated output turbulence is used with the methods of Sec. III to produce the linear growth/ damping rates and amounts of nonlinear energy transfer, which are then compared with the values that generated the turbulence.

We use a contrived 1-D model that mimics the 2-D polarization drift nonlinearity. From the Hasegawa-Mima equation, ${ }^{20}$ the polarization drift nonlinearity is given by
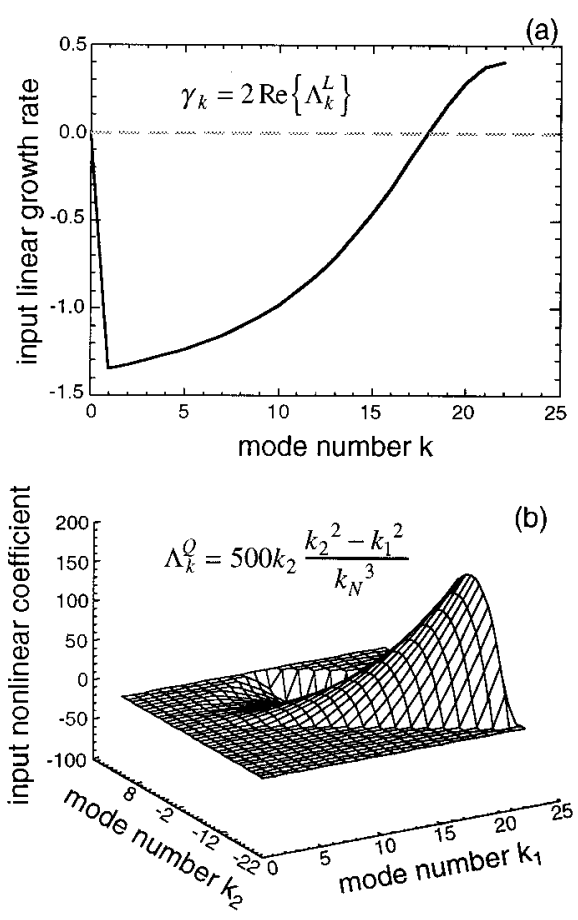

FIG. 1. Input linear growth rate (a) and nonlinear coupling coefficient (b) used for generating the pure 1-D simulated, fully developed turbulence data.

$$
\Lambda_{k}^{Q}=C_{\text {pol }}\left(k_{x} k_{y_{1}}-k_{y} k_{x_{1}}\right)\left[\left(k_{x_{2}}^{2}+k_{y_{2}}^{2}\right)-\left(k_{x_{1}}^{2}+k_{y_{1}}^{2}\right)\right] .
$$

For our 1-D model we use a coupling coefficient given by

$$
\Lambda_{k}^{Q}=C_{\mathrm{pol}}^{\prime}\left(k_{1}-k\right)\left(k_{2}^{2}-k_{1}^{2}\right) \text {. }
$$

Comparison with the polarization drift nonlinearity suggests that the 1-D model represents a spectrum whose radial width $\left(\Delta k_{x}\right)$ is much narrower than the poloidal width $\left(\Delta k_{y}\right)$. However, it should be cautioned that this model is ultimately unphysical as a projection of the polarization drift nonlinearity because of the tendency of the polarization drift nonlinearity to isotropize any spectrum with $\Delta k_{x} \ll \Delta k_{y}$.

Figure 1 illustrates the input linear growth rate and the input nonlinear transfer coefficient. It shows that for saturation of the fluctuations, nonlinear energy transfer must go from linearly unstable modes at high wave number to damped or dissipative modes at low wave number. The simulated output fluctuation is fully saturated with 45 mode numbers, as indicated by Fig. 2(a). The peak in the fluctuation power spectrum at intermediate wave number [Fig. 2(b)] is formed by the polarization nonlinear energy cascade balanced against linear growth and dissipation.

With the Ritz method and the modified Ritz method of Sec. III, we estimate the linear growth/damping rate and the nonlinear coupling coefficient. As shown in Figs. 3(c) and 3(d), the modified Ritz method exactly recovers the input characteristics of the simulation, but the Ritz method [Figs. 3(a) and 3(b)] fails to reproduce the true values at almost all wave numbers.

For this test, there are no nonideal fluctuations in the data. The assumption of stationarity is not crucial here and the failure of the Ritz method is caused only by the assump- 

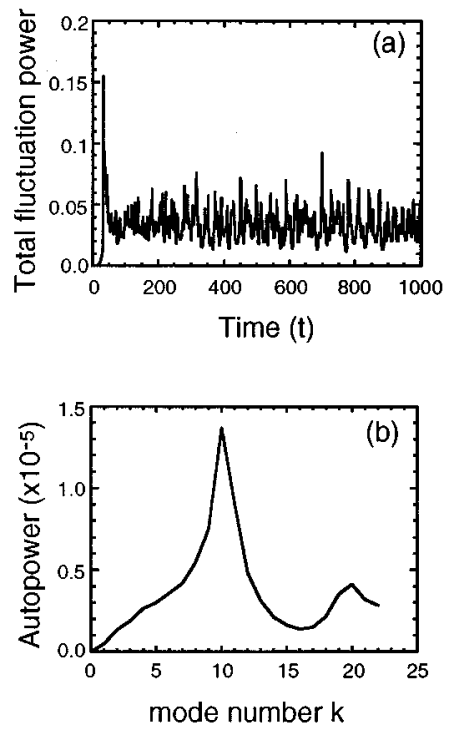

FIG. 2. (a) Evolution of total fluctuation power showing saturation of turbulence data. (b) Power spectrum of the pure 1-D simulation data.

tion that replaces the fourth-order moment with the square of the second-order moment. It indicates that the fourth-order cumulant is mathematically necessary to perfectly describe the moments of our governing equation (1). Fourth-order cumulants are non-negligible in this simulation because finite spectral resolution, exacerbated by one-dimensionality, so limits the spectral coupling that phase-coherent triplets dominate the evolution. This violates the random phase assumption that allows fourth-order moments to be expressed as products of second-order moments.

This simulation experiment confirms that the 1-D algorithm of the modified Ritz method (or the Ritz method with all fourth-order cumulants) fully recovers the linear growth rate and the rates of nonlinear energy transfer for pure 1-D turbulence data. However, pure 1-D turbulence is ultimately unphysical. That is, the nature of turbulence in magnetically confined high-temperature plasmas is generally thought to be two-dimensional (2-D) because the parallel wavelength is sufficiently long. This motivates 2-D simulation experiments.

\section{B. Test of the procedure with 2-D turbulence data}

We simulate the turbulence of a magnetically confined high-temperature plasma with the Terry-Horton model, which is a two-dimensional single-field nonlinear model ${ }^{21-23}$ described by

$$
\begin{aligned}
\frac{\partial \widetilde{n_{k}}}{\partial t}= & D k_{y}^{2} \tilde{n_{k}}-i v_{D} k_{y} \tilde{n_{k}}-\nu_{\mathrm{eff}, i} \tilde{n_{k}}-\mu k^{4} \tilde{n_{k}}-N_{k}^{(\mathrm{pol})} \\
& -N_{k}^{(\widetilde{E} \times B)}
\end{aligned}
$$

where

$$
\begin{aligned}
& N_{k}^{(\widetilde{E} \times B)}=\frac{i}{2} L_{n} D \sum_{k^{\prime}} \vec{k} \times \vec{k}^{\prime} \hat{z}\left[k_{y}^{\prime}-\left(k_{y}-k_{y}^{\prime}\right)\right] \tilde{n_{k^{\prime}}} \tilde{n}_{k-k^{\prime}}, \\
& N_{k}^{(\mathrm{pol})}=\frac{1}{2} \rho_{s}^{3} C_{s} \sum_{k^{\prime}} \vec{k} \times \vec{k}^{\prime} \cdot \hat{z}\left[\left(k_{\perp}-k_{\perp}^{\prime}\right)^{2}-k_{\perp}^{\prime 2}\right] \tilde{n_{k}} \tilde{n}_{k-k^{\prime}} .
\end{aligned}
$$
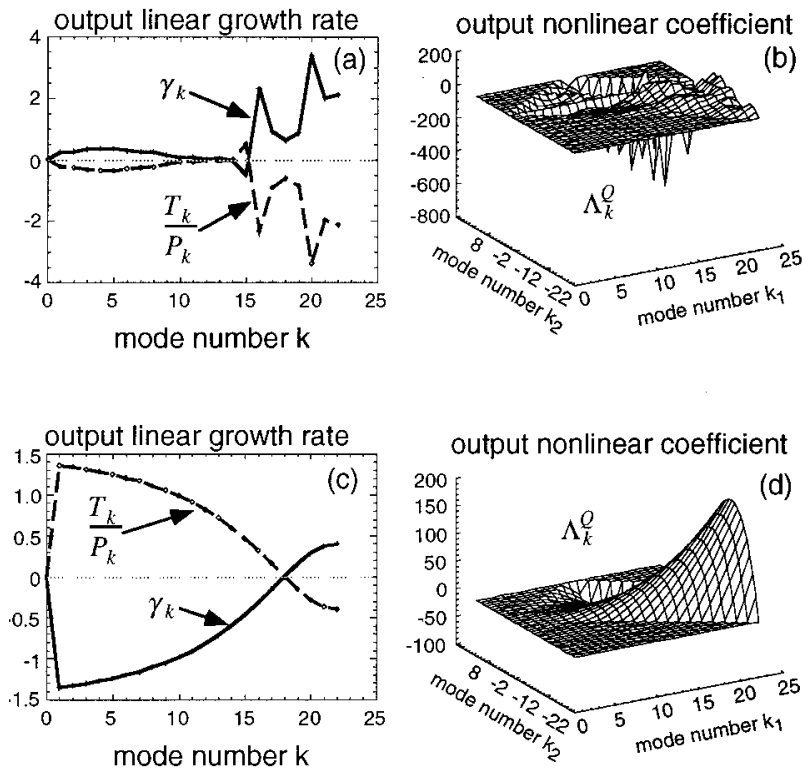

FIG. 3. Output linear growth rate (a) and linear coupling coefficient (b) estimated by the Ritz method, and output linear growth rate (c) and nonlinear coupling coefficient (d) estimated by the modified Ritz method.

In this model, $\tilde{n}$ is the fluctuating ion density, $v_{D}=\left(c T_{e} /\right.$ $e B) L_{n}{ }^{-1}$ is the diamagnetic drift velocity, $D=\epsilon^{1 / 2} v_{D}^{2}(1$ $\left.+3 \eta_{e} / 2\right) / \nu_{\text {eff }, e}$ is a negative diffusivity describing the destabilization of the dissipative trapped electron (DTE) modes by electron collisions, $L_{n}$ is the density gradient scale length, $\rho_{s}=\left(c T_{e} / e B\right) / C_{s}$ is the ion gyroradius evaluated at the electron temperature, $C_{s}=\left(T_{e} / m_{i}\right)^{1 / 2}$ is the ion sound speed, and $\mu$ is the coefficient of hyperviscosity, introduced to model strong damping at high wave number and to realistically cut off the maximum wave number in the simulation to finite values.

The first nonlinearity $\left(N_{k}^{(\mathrm{pol})}\right)$ appearing in Eq. (19) is the polarization drift nonlinearity, and arises from $n_{0} \boldsymbol{\nabla} \cdot \vec{v}_{p}^{(1)}$, where $\vec{v}_{p}^{(1)}=B_{0}^{-1}\left(m_{i} c / e\right) \hat{z} \times \vec{v}_{E} \cdot \boldsymbol{\nabla} \vec{v}_{E}$ is the nonlinear polarization drift. The second nonlinearity $\left(N_{k}^{(\widetilde{E} \times B)}\right)$ is the $\widetilde{E} \times B$ nonlinearity, arising from $\vec{v}_{E} \cdot \nabla \widetilde{n}_{e}$, where $\vec{v}_{E}=-(c)$ $\left.B_{0}\right) \boldsymbol{\nabla} \phi \times \hat{z}$ is the $\widetilde{E} \times B$ drift, and $\widetilde{n}_{e}$ is the nonadiabatic electron density, which, from the electron response, is related back to the potential, and, through quasineutrality, to the ion density.

To generate saturated 2-D turbulence data, we input values for the physical variables $\left(D, v_{D}, \nu_{\text {eff }, i}, \mu, L_{n}, C_{s}\right.$, $\rho_{s}$ ) and numerically integrate Eq. (19), starting from Gaussian random spectra, to get the time evolution of the fully developed wave number spectra. We generate more than 2000 realizations (which are used for ensemble averaging) at each wave number $k_{x}$ and $k_{y}$, where each realization consists of two samples: one at time $t$ and the other at time $t+\tau$, where $\tau$ is a delay time between the samples and is much smaller than the eddy turnover time. Each realization must be separated from the next by a time longer than the eddy turnover time to get statistically independent realizations. This sampling method mimics the experimental turbulent data measured via beam emission spectroscopy (BES). ${ }^{9}$ A 

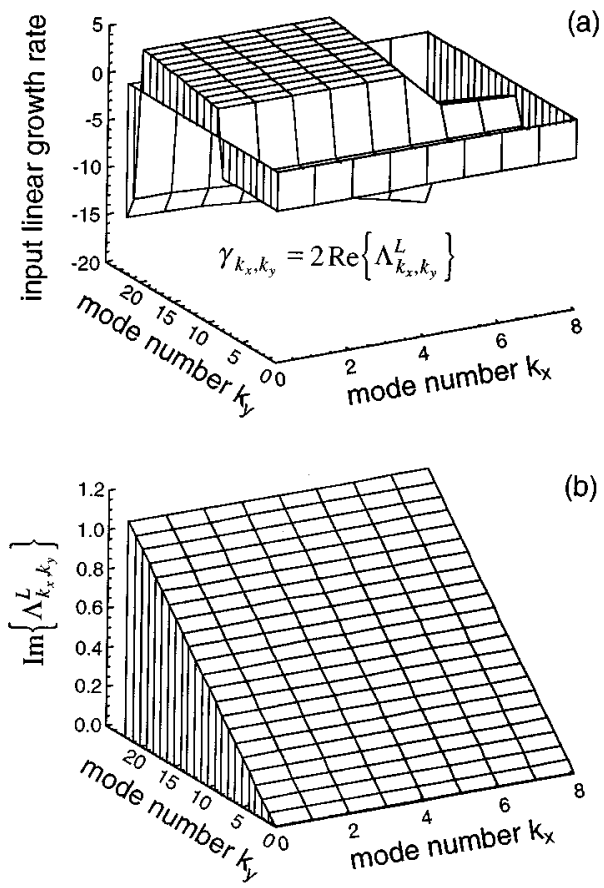

FIG. 4. True 2-D linear growth rate (a) and dispersion relation (b) as the input linear characteristics of the 2-D simulation.

total of 2000 realizations is used for the results discussed herein. Tests with more realizations indicate that fully stationary results are obtained with these 2000 realizations, and further ensemble averaging does not change the derived results.

As shown in Fig. 4, the unstable modes are chosen at intermediate $k$ with damping at high and low $k$. The imaginary part of the input linear transfer function, representing the linear fluctuation frequency (or dispersion relation), is proportional to the normalized poloidal mode number $k_{y}$. Here $17 \times 45$ mode numbers and ten million time steps with $\Delta t=0.0001$ for a total run time of $T=1000$ (where the eddy turnover time is between 0.5 and 1) are used to get the time evolution of fully developed turbulence. Figure 5 shows that the power spectrum is anisotropic with respect to the peak, and the peak of the fluctuation power is near $k_{x}=0$ and $k_{y}=9$, instead of at the $k_{y}=20$ mode which is the most unstable mode, as shown in Fig. 4(a).

To recover the 2-D input characteristics of this simulated dataset, we generalize the governing equation (1) for twodimensional turbulence, which is then

$$
\begin{aligned}
\frac{\partial \varphi_{k_{x}, k_{y}}(t)}{\partial t}= & \Lambda_{k_{x}, k_{y}}^{L} \varphi_{k_{x}, k_{y}}(t) \\
& +\frac{1}{2} \sum_{\substack{k_{x_{1}}, k_{x_{2}} \\
k_{x}=k_{x_{1}}+k_{x_{2}}}} \sum_{\substack{k_{y}=k_{y_{1}}, k_{y_{2}}+k_{y_{2}} \\
k_{k_{x}}, k_{y}}}^{Q}{ }^{Q} \\
& \times \varphi_{k_{x_{1}}, k_{y_{1}}}(t) \varphi_{k_{x_{2}}, k_{y_{2}}}(t) .
\end{aligned}
$$

By implementing the same procedure (i.e., $Y_{k}, X_{k}, X_{k_{1}}$,

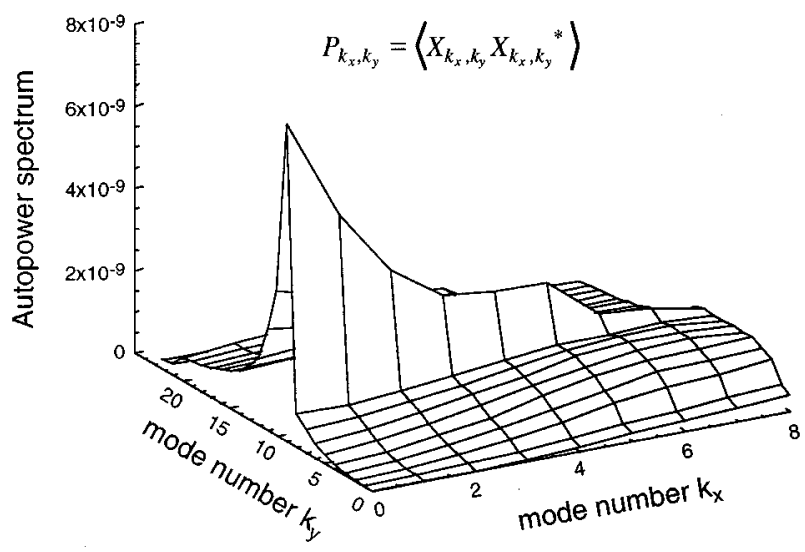

FIG. 5. Autopower spectrum of the 2-D simulated turbulence data.

$X_{k_{2}}, L_{k}, Q_{k}^{k_{1}, k_{2}}$, and $\Sigma_{k_{1} \geqslant k_{2}}$ are directly replaced by $Y_{k_{x}, k_{y}}, X_{k_{x}, k_{y}}, X_{k_{x_{1}}, k_{y_{1}}}, X_{k_{x_{2}}, k_{y_{2}}}, L_{k_{x}, k_{y}}, Q_{k_{x}, k_{y}}^{k_{x_{1}}, k_{x_{2}}, k_{y_{1}}, k_{y_{2}}}$, and $\frac{1}{2} \sum_{k_{x_{1}}}, k_{x_{2}} \quad \sum_{k_{1}}, k_{y_{2}} \quad$ ) of Sec. III on this 2-D governing $k_{x}=k_{x_{1}}+k_{x_{2}} \quad k_{y}=k_{y_{1}}+k_{y_{2}}$

equation (which we refer to as the 2-D algorithm), we can estimate the 2-D input linear growth rate and linear dispersion relation.

The 2-D algorithm of the Ritz method reasonably estimates the input linear dispersion relation, but does not accurately recover the input linear growth rate, as shown in Fig. 6. There are significant quantitative differences between the
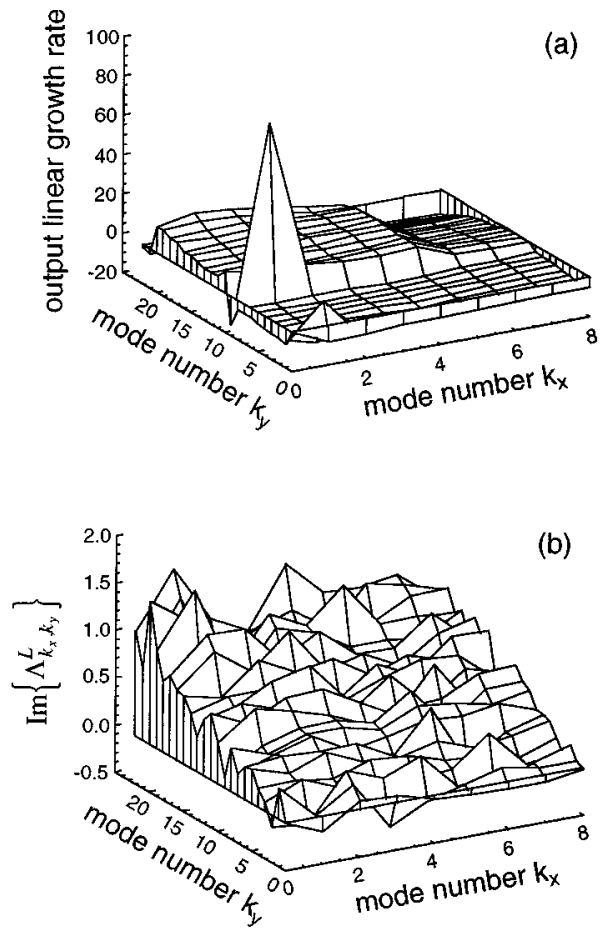

FIG. 6. The estimated 2-D linear growth rate (a) and dispersion relation (b) computed with the 2-D algorithm of the Ritz method. 

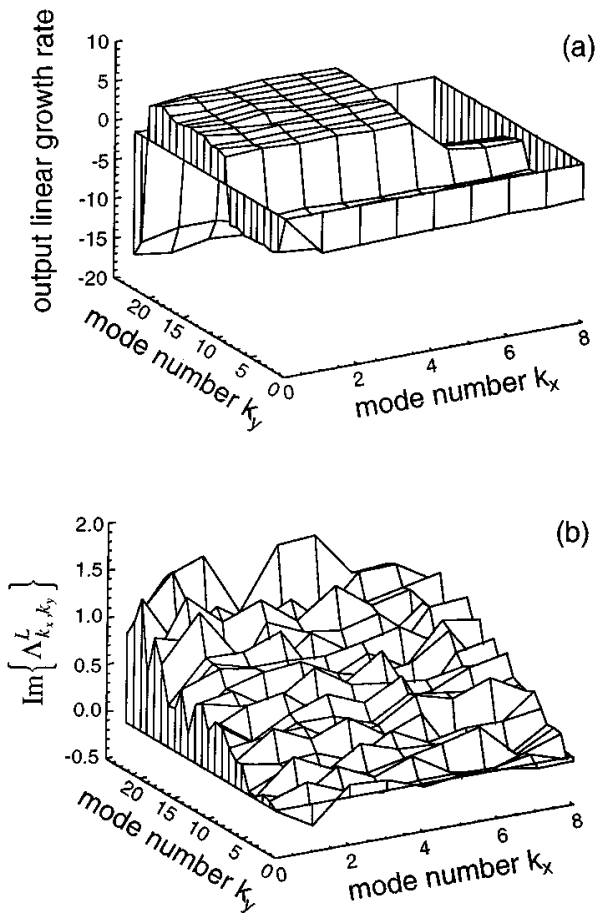

FIG. 7. The estimated 2-D linear growth rate (a) and dispersion relation (b) computed with the 2-D algorithm of the modified Ritz method.

output and input linear growths in Fig. 6 even if one ignores the single spike (which occurs due to a local strong variation in the fourth-order moment). For example, the amplitudes of the output linear growth rate at most wave numbers are twice as large as the input values (e.g., maximum positive growth rate is about 10 , in contrast to 5 in input from the $2-\mathrm{D}$ model). Likewise, the damping rates at low $k_{y}$ ( $<10$ or so) are larger than the damping rates at high $k_{y}(\geqslant 21)$ on the output, whereas the opposite holds for the input.

However, the 2-D algorithm of the modified Ritz method reproduces both the linear dispersion relation and the linear growth rate in a more satisfactory way, as shown in Fig. 7, where the output growth rates are almost equal to the input values at all wave numbers. In addition, the 2-D algorithm of the Ritz method with the fourth-order moment (instead of the square of the second-order moment as prescribed in the original Ritz formulation) also produces the same result [Fig. 7(a)] as that of the modified Ritz method. This again suggests that the fourth-order moment is mathematically necessary to perfectly describe the moments of our governing equation (20), especially for simulation data. This violation of the random phase assumption, which allows the fourthorder moment to be expressed as solely the products of the second-order moment, is possibly due to finite spectral resolution and the finite grid size of the simulation, which forces each phase of wave number spectra to be strongly coupled and thus produces significant fourth-order coupling.

From these results, it is found that for "clean" turbulence data, the modified Ritz method is equivalent to the Ritz method with the fourth-order moment, provided that the turbulence is fully developed. However, the advantage of the modified Ritz method over the Ritz method is improved
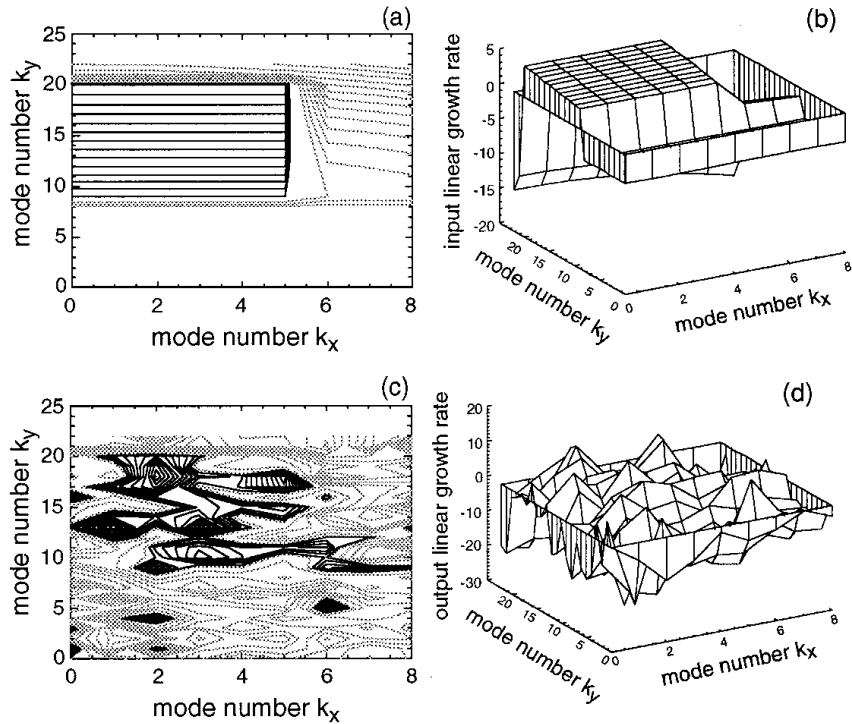

(d)
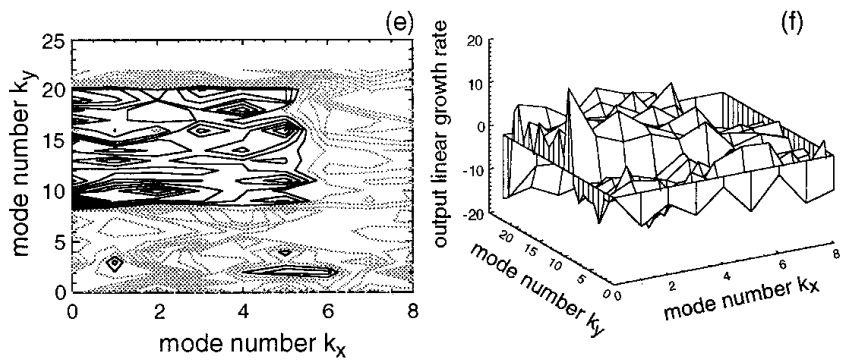

FIG. 8. The true linear growth rate [(a) and (b)] and the estimated 2-D linear growth rate by the Ritz method with the fourth-order moment [(c) and (d)] and by the modified Ritz method [(e) and (f)] when the 2-D simulated turbulence data are contaminated with Gaussian noise.

noise rejection when nonideal data is encountered.

To examine the influence of noise on this analysis, we apply the 2-D algorithm of the modified Ritz method and that of the Ritz method with the fourth-order moment to the 2-D simulated turbulence data with an added Gaussian random noise. Figure 8 shows the true 2-D linear growth rate of the 2-D simulation and the estimated 2-D linear growth rate by the Ritz method with the fourth-order moment and the modified Ritz method when the 2-D simulated turbulence data are contaminated with this $-30 \mathrm{~dB}$ Gaussian random noise. Here dark lines represent positive contours (linearly growing) and gray lines represent negative contours (linearly damping) in contour plots. As shown in Figs. 8(c) and 8(d), the 2-D algorithm of the Ritz method with the fourth-order moment produces a 2-D linear growth rate, which is strongly damped in regions of actual growth. This is due to the nonideal power not described by the governing equation (20). In contrast, the 2-D algorithm of the modified Ritz method more reasonably reproduces the 2-D linear growth rate [Figs. $8(\mathrm{e})$ and $8(\mathrm{f})$ ] and thus reduces noise effects by more accurately extracting the turbulent autopower spectrum via higher moments.

Note that the $-30 \mathrm{~dB}$ of Gaussian random noise is defined relative to the average fluctuation power and thus can 
be dominant in the region of low-amplitude fluctuation (i.e., noise $\gg$ fluctuation power where the power is small). In addition, the effect of noise is exacerbated in the 2-D case due to the strong influence of the fourth-order moments, which are noise sensitive. In contrast, when we apply the 1-D algorithm of the modified Ritz method to 1-D experimental data with up to $-10 \mathrm{~dB}$ of Gaussian random noise (relative to the average power), the modified Ritz method keeps the linear growth rate within $10 \%$ of the value determined without the added noise, indicating that the off-diagonal fourthorder terms are negligible in the experimental turbulence data.

Based on this and other 2-D simulation experiments, the 2-D algorithm of the modified Ritz method is found to produce reliable estimates of the 2-D linear growth rate and the resulting rate of nonlinear energy transfer with acceptable noise rejection.

\section{APPLICATION TO EXPERIMENTAL TURBULENCE DATA}

In practical cases dealing with experimental density fluctuation measured via BES or other spatial sampling fluctuation measurements, only 1-D, radially averaged turbulence data rather than a complete 2-D turbulence dataset is typically available. To develop an appropriate form of a 1-D algorithm to apply to the experimental 1-D radially averaged data, we test the 1-D algorithm of the modified Ritz method with 1-D fluctuation data derived from the full 2-D simulated dataset.

The experimental density fluctuations are measured via BES in the core plasma region of the Tokamak Fusion Test Reactor (TFTR). ${ }^{24}$ This density fluctuation is given by

$$
\begin{aligned}
\widetilde{n}\left(\omega_{\mathrm{LAB}}, x, y\right)= & \int_{-k_{\mathrm{N}}}^{k_{\mathrm{N}}} \int_{-k_{\mathrm{N}}}^{k_{\mathrm{N}}} \widetilde{n}\left(\omega_{\mathrm{LAB}}, k_{x}, k_{y}\right) \\
& \times T\left(k_{x}, k_{y}\right) e^{i \bar{k} \cdot \bar{r}} d k_{x} d k_{y},
\end{aligned}
$$

where $k_{\mathrm{N}}$ is the Nyquist wave number, $x$ and $y$ represent the radial and poloidal direction, and $T\left(k_{x}, k_{y}\right)$ is a transfer function that is determined by the finite sample volume. The frequency spectra observed in the laboratory frame in TFTR are found to be completely dominated by rotation-induced Doppler shifts of the local $S(k)$ spectrum. ${ }^{25}$ This dominance of the Doppler shift in the measured spectra allows a study of the nonlinear coupling between unstable modes at various $k$ through a bispectral analysis of the frequency spectra. Given this bulk plasma rotation, we can assign a poloidal wave number value to the observed frequencies as a first approximation, that is,

$$
\widetilde{n}\left(x, k_{y}\right)=\int_{-k_{\mathrm{N}}}^{k_{\mathrm{N}}} \widetilde{n}\left(k_{x}, k_{y}\right) T\left(k_{x}, k_{y}\right) e^{i k_{x} x} d k_{x} .
$$

To simulate this 1-D experimental density fluctuation data measured via BES, we average (inverse Fourier transform in the radial wave number space at $x=0$ ) the 2-D simulated turbulence data over radial wave number $\left(k_{x}\right)$.

Using this averaged 2-D simulated turbulent data, we can apply the procedure for one-dimensional turbulence data
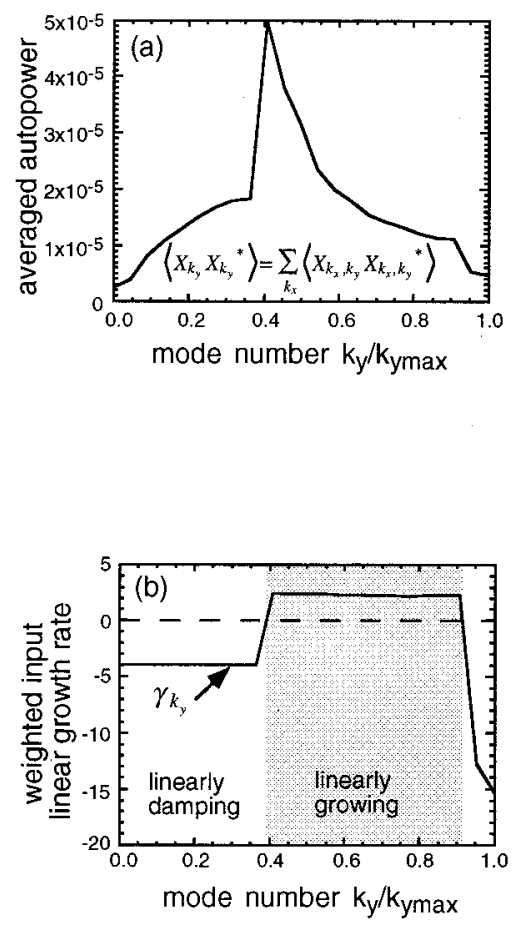

FIG. 9. (a) The averaged 2-D autopower spectrum over the radial wave number. (b) The weighted true input linear growth rate when the 2-D simulated turbulence data is reduced to averaged 1-D data.

in order to characterize the weighted (1-D) linear growth rate of input variables of the 2-D simulated turbulence data.

\section{A. Test of the modified Ritz method with averaged 2-D data}

To examine how well the 1-D algorithm of the modified Ritz method interprets the nonlinear characteristics of the 1-D experimental fluctuation data measured via BES, we apply the 1-D algorithm to the 2-D simulated turbulence data, which is averaged over radial wave number to simulate 1-D experimental turbulence data.

The test of the modified Ritz method is performed with the averaged 2-D simulation data, which is the same data used for the 2-D algorithm in Sec. IV B. In this averaging process, we assume a unity radial transfer function, $T\left(k_{x}\right)=1$ (note that the influence of the radial transfer function is discussed in Ref. 26). Figure 9 shows the weighted autopower spectrum and input linear growth rate for the averaged data, where again the resulting peak fluctuation power is shifted by the nonlinear interactions to regions away from the most linearly unstable modes. In this test, we try to recover the weighted linear growth rate [Fig. 9(b)] of the 2-D simulation given by

$$
\gamma_{k_{y}}=\frac{\sum_{k_{x}} \gamma_{k_{x}, k_{y}}\left\langle X_{k_{x}, k_{y}} X_{k_{x}, k_{y}}^{*}\right\rangle}{\left\langle X_{k_{y}} X_{k_{y}}^{*}\right\rangle},
$$

by using the averaged 2-D data $\left[Y_{k}\left(=\Sigma_{k_{x}} Y_{k_{x}, k_{y}} \Delta k_{x}\right), X_{k}\right.$ ( $\left.\left.=\Sigma_{k_{x}} X_{k_{x}, k_{y}} \Delta k_{x}\right)\right]$ and the modified Ritz method.

The result of this analysis is shown in Fig. 10, where the solid dotted line represents the estimated linear growth rate and the gray line represents the rate of nonlinear energy 


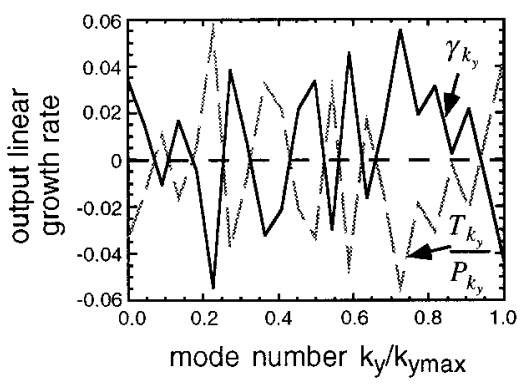

FIG. 10. The derived linear growth rate computed with the 1-D algorithm of the modified Ritz method from the averaged 2-D simulated turbulence data.

transfer as given by the power flow, Eq. (4). As shown in Fig. 10, the modified Ritz method does not recover the weighted input characteristics of the 2-D simulation data [i.e., the $\gamma_{k_{y}}$ in Fig. 10 does not reproduce the input $\gamma_{k_{y}}$ in Fig. 9(b)]. This suggests that the bispectrum calculation in the modified Ritz method does not recover the three-wave coupling information from the averaged 2-D simulation data. In identifying the cause of this failure, it should be noted that the fourth-order moment can be replaced with the square of the second-order moment in the averaged 2-D simulation data with no significant change in the resulting $\gamma_{k_{y}}$ output. This contrasts with the finding that the deduced $\gamma_{k_{x}, k_{y}}$ was very sensitive to the use of the 2-D complete fourth-order moments, and thus suggests that the averaging process validates the random phase assumption by suppressing the higher-order phase relationships. This statement is also applicable to the 1-D experimental data.

From this, it is found that the phase of the averaged wave number spectra is less coherent and loses information on the three-wave coupling (bispectrum) through the averaging process. This lack of information of three-wave coupling results in linear growth rates that are two orders of magnitude smaller than the weighted input linear growth rate.

\section{B. Nonresonant 1-D algorithm for 1-D experimental turbulence data}

From Eq. (18), we may approximate the fourth-order moment with the square of the second-order moment because of the lower coherence of the phase of the averaged 2-D turbulence data (as discussed in Sec. V A). The linear growth rate is then estimated to be

$$
\gamma_{k_{y}} \approx \frac{1}{\tau} \frac{\sum_{\substack{k_{y_{1}} \geqslant k_{y_{2}} \\ k_{y}=k_{y_{1}}+k_{y_{2}}}} \frac{\left|\left\langle X_{k_{y_{1}}} X_{k_{y_{2}}} X_{k_{y}}^{*}\right\rangle\right|^{2}-\left|\left\langle X_{k_{y_{1}}} X_{k_{y_{2}}} Y_{k_{y}}^{*}\right\rangle\right|^{2}}{\left\langle\left|X_{k_{y_{1}}} X_{k_{y_{2}}}\right|^{2}\right\rangle}}{\left\langle\beta_{k} \beta_{k}^{*}\right\rangle-\sum_{\substack{k_{y_{1}} \geqslant k_{y_{2}} \\ k_{y}=k_{y_{1}}+k_{y_{2}}}} \frac{\left|\left\langle X_{k_{y_{1}}} X_{k_{y_{2}}} X_{k_{y}}^{*}\right\rangle\right|^{2}}{\left\langle\left|X_{k_{y_{1}}} X_{k_{y_{2}}}\right|^{2}\right\rangle}} .
$$

From this equation, it is seen that the linear growth or damping is entirely determined by the auto- and cross-bispectra. Hence, the exact evaluation of these bispectra is important in obtaining optimal reconstructions of input variables from the averaged phase. To account for broadening of the bispectra induced by the radial averaging process and include all possible three-wave coupling information at wave number $k_{y}$, we consider a modified 1-D governing equation that includes all nonresonant nonlinearities to be applied to the radially averaged data. The modified governing equation for this nonresonant 1-D algorithm is taken to be

$$
\frac{\partial \varphi_{k_{y}}}{\partial t}=\Lambda_{k_{y}}^{L} \varphi_{k_{y}}+\sum_{k_{y_{1}}, k_{y_{2}}} \Lambda_{k_{y}}^{Q} \varphi_{k_{y_{1}}} \varphi_{k_{y_{2}}} .
$$

By implementing the same procedure as the modified Ritz method without the off-diagonal terms of the fourth-order moments on this new governing equation, we can evaluate the weighted linear growth rate for the averaged 2-D simulated turbulence data.

The main advantage of this approach is that the values of the third-order cumulants are zero if there is no phase relation in the three-wave coupling. This, in turn, gives better estimates of the bispectra and correspondingly better estimates of the $\gamma_{k_{y}}$.

With the previous 1-D simulation obtained by reduction of the 2-D simulated data of Sec. V A (whose weighted linear growth rate was not recovered by the original 1-D algorithm), the new nonresonant 1-D algorithm reconstructs the input linear growth rate and nonlinear coupling coefficients for the simulated data within the same order of magnitude and with growth and damping occurring at the proper wave numbers, as indicated in Fig. 11(b). In Figs. 11(b) and 12(b), the thick gray lines are smoothed versions of the thin lines (which are the actual output values). The radial wave number spectrum [shown in Fig. 12(a)] and the amplitude of net flow of the nonlinear energy [Fig. 12(b)], given by

$$
\sum_{k_{y_{1}}, k_{y_{2}}} \frac{\left|\left\langle X_{k_{y_{1}}} X_{k_{y_{2}}} X_{k_{y}}^{*}\right\rangle\right|^{2}-\left|\left\langle X_{k_{y_{1}}} X_{k_{y_{2}}} Y_{k_{y}}^{*}\right\rangle\right|^{2}}{\left\langle\left|X_{k_{y_{1}}} X_{k_{y_{2}}}\right|^{2}\right\rangle},
$$

indicate that there is significant power at all radial wave numbers and they are all strongly coupled. Because there is strong three-wave coupling at all spatial scales (all $k$ ), the radial averaging of the spectrum causes a more extensive, nonreversible loss of phase information. This, in turn, limits the accuracy of the reconstruction. Observations from many simulation experiments indicate that better results are obtained for spectra that are highly localized (i.e., all the fluctuation power is concentrated in a narrow range of the radial wave number space, $k_{x}$ ). In this case, the spectra are nearly "one-dimensional" and radial averaging destroys relatively little phase information. However, for the very broad spectra (larger range in $k_{x}$ ) shown in this simulation [Fig. 12(a)], more phase information is progressively destroyed by radial averaging. Similarly, in simulated data generated solely by the $\widetilde{E} \times B$ nonlinearity, with its anisotropic transfer, more phase information is lost in radial averaging than it is in simulations generated solely by the isotropic polarization drift nonlinearity.

In general, the nonresonant 1-D algorithm reconstructs the linear growth rates and nonlinear coupling coefficients of 

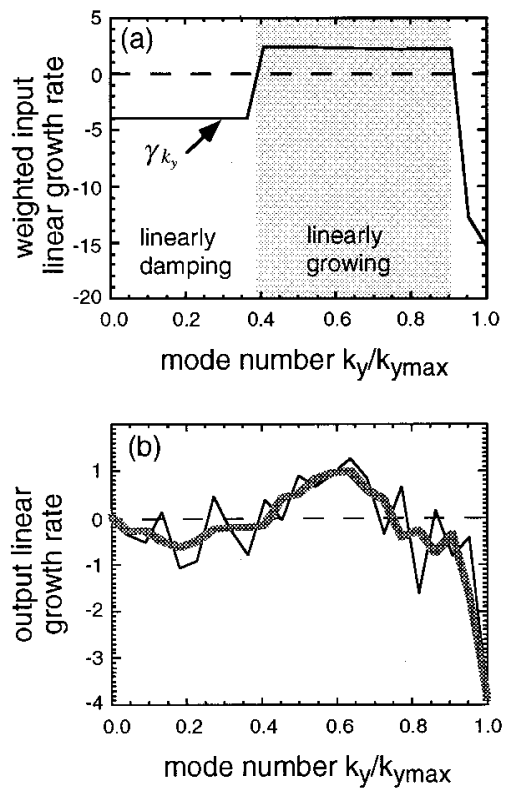

FIG. 11. (a) The weighted input linear growth rate of the averaged 2-D simulation. (b) The derived linear growth rate with the nonresonant 1-D algorithm from the averaged 2-D simulation data.

the averaged 2-D simulation data in an approximate but much more satisfactory manner when the width of the radial wave number spectrum is comparable to or less than that of the poloidal spectrum. How well it quantitatively reproduces the input linear growth rates will depend on the details of the spectra and the underlying turbulence.

Since only the nonresonant 1-D algorithm reproduces, at least qualitatively, the radially averaged characteristics of turbulence described by the original 2-D governing equation, it is the algorithm that should be applied to analysis of the 1-D tokamak plasma turbulence data. While quantitative results from this analysis will only be relevant when used in comparison to full 2-D theoretical models of the turbulence in machines such as TFTR, the qualitative trends in the deduced $\gamma_{k}$ 's should accurately reproduce regions of relative growth and stability.

\section{SUMMARY AND CONCLUSION}

A new procedure for experimentally estimating the energy cascade and linear growth/damping rate of fully developed turbulence has been developed to study the physics of plasma turbulence. Application of this new procedure to the reconstruction of turbulent spectra eliminates the unphysically large damping rates observed using the original method of Ritz et al. It employs stationarity or statistical ergodicity of the turbulence to eliminate the effects of noise and fluctuations not described by the governing equation, and can include the fourth-order moment to avoid closure approximations as needed.

To examine the validity of the procedure, a 2-D algorithm has been implemented and tested on simulated, fully developed 2-D turbulent data from a 2-D trapped-particle fluid model code, which has polarization drift and $\widetilde{E} \times B$ nonlinearities. Also, a 1-D algorithm has been tested on the av-
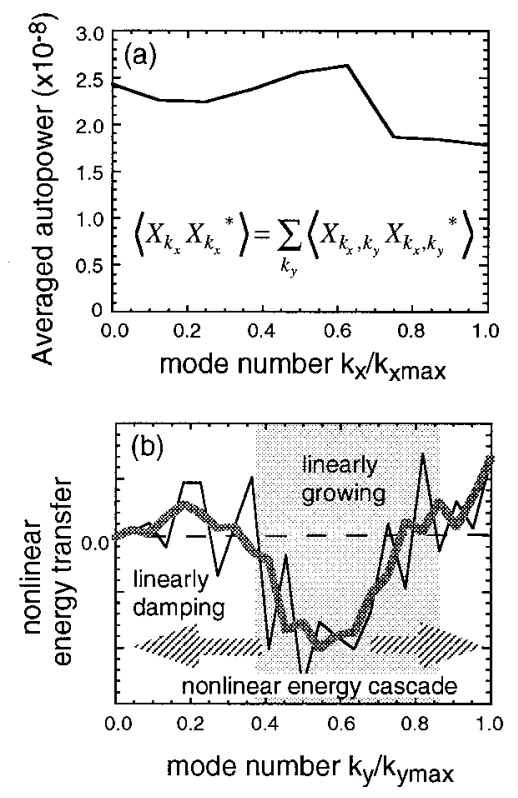

FIG. 12. (a) The averaged 2-D autopower spectrum over the poloidal wave number. (b) The net flow of nonlinear energy, $T_{\text {out }}-T_{\text {in }}$.

eraged (inverse Fourier transformed) 2-D simulation data to estimate how well a standard 1-D algorithm can extract reasonable physical results from 1-D experimental data. The results show that the 2-D algorithm can fully and accurately reproduce the input characteristics of the 2-D simulation data with good noise rejection, but the standard 1-D algorithm does not, in general, reproduce the input variables, especially from the averaged 2-D simulation data that has significant fluctuation power above half the Nyquist wave number for the averaged axis (i.e., $\Delta k_{x}>k_{\mathrm{N}} / 2$ ).

We have developed a nonresonant 1-D algorithm that includes nonresonant wave numbers in the 1-D governing equation to include three-wave coupling information from the averaged phase. This nonresonant 1-D algorithm is able to qualitatively reproduce the input growth rate spectrum from the simulated, 1-D experimental fluctuation data when the width of the radial wave number spectrum is comparable to or less than that of the poloidal spectrum. This criterion is usually satisfied with density fluctuation data measured via BES.

The work in this paper assumes that the saturated turbulence may be described adequately by a one-field model. In reality, tokamak turbulence is characterized by multifield fluctuations (density, temperature, electric potential, etc.). We have made the assumption that the observation of one of these fields (density) is sufficient to characterize the nonlinear behavior of the system. This assumption is reasonable for saturated turbulence. However, it has not been rigorously tested to date. In the future, further simulation tests will be performed using more sophisticated, multifield models that should allow the role of multiple fields to be evaluated.

Finally, the nonresonant 1-D algorithm described here will be applied to density fluctuation data measured via beam emission spectroscopy (BES) for quantifying the linear growth/damping rate and the resulting amount of nonlinear 
energy transfer in the core of TFTR. Such experimental measurements and analysis can be useful for a direct comparison with full 2-D theoretical models.

\section{ACKNOWLEDGMENTS}

This work was supported by the U.S. Department of Energy Grant No. DE-FG02-89ER53296 and Contract No. DEAC02-76-CHO-3078.

${ }^{1}$ Ch. P. Ritz and E. J. Powers, Physica D 20, 320 (1986).

${ }^{2}$ Ch. P. Ritz, E. J. Powers, R. W. Miksad, and R. S. Solis, Phys. Fluids 31, 3577 (1988).

${ }^{3}$ Ch. P. Ritz, E. J. Powers, and R. D. Bengtson, Phys. Fluids B 1, 153 (1989).

${ }^{4}$ M. Rosenblatt, Proceedings of the Symposium of Time Series Analysis (Wiley, New York, 1963), p. 125.

${ }^{5}$ Y. C. Kim and E. J. Powers, Phys. Fluids 21, 1452 (1978).

${ }^{6}$ Y. C. Kim and E. J. Powers, IEEE Trans. Plasma Sci. PS-7, 120 (1979).

${ }^{7}$ S. Assadi, S. C. Prager, and K. L. Sidikman, Phys. Rev. Lett. 69, 281 (1992).

${ }^{8}$ C. Hidalgo, E. Sanchez, T. Estrada, B. Branas, and Ch. P. Ritz, Phys. Rev. Lett. 71, 3127 (1993).

${ }^{9}$ R. J. Fonck, P. A. Duperrex, and S. F. Paul, Rev. Sci. Instrum. 61, 3487 (1990).

${ }^{10}$ S. F. Paul, N. Bretz, R. D. Durst, R. J. Fonck, E. Mazzucato, and R. Nazikian, Phys. Fluids B 4, 2922 (1992).

${ }^{11}$ P. M. Schoch, J. G. Schatz, T. P. Crowley, K. A. Conner, A. Carnevali, J. C. Forster, J. F. Lewis, G. A. Hallock, and R. L. Hickok, Rev. Sci. Instrum. 9, 1646 (1988).

${ }^{12}$ E. Mazzucato and R. Nazikian, Plasma Phys. Controlled Fusion 33, 261 (1991).
${ }^{13}$ J. D. Callen, Phys. Fluids B 4, 2142 (1992).

${ }^{14}$ R. W. Miksad, F. L. Jones, and E. J. Powers, Phys. Fluids 26, 1402 (1983).

${ }^{15}$ S. Elgar and R. T. Guza, J. Fluid Mech. 161, 425 (1985).

${ }^{16}$ M. D. Millionshchikov, Dokl. Akad. Nauk SSSR 32, 611 (1941).

${ }^{17}$ C. L. Nikias and M. R. Raghuveer, Proc. IEEE 75, 869 (1987).

${ }^{18}$ B. Harris, Spectral Analysis of Time Series (Wiley, New York, 1967), p. 153.

${ }^{19}$ M. B. Priestley, Spectral Analysis and Time Series (Academic, London, 1981).

${ }^{20}$ A. Hasegawa and K. Mima, Phys. Fluids 21, 87 (1978).

${ }^{21}$ D. E. Newman, P. W. Terry, and P. H. Diamond, Phys. Fluids B 4, 599 (1992).

${ }^{22}$ D. E. Newman, P. W. Terry, P. H. Diamond, and Y. M. Liang, Phys. Fluids B 5, 1140 (1993).

${ }^{23}$ P. W. Terry and W. Horton, Phys. Fluids 25, 491 (1982).

${ }^{24}$ J. Strachan, M. Bitter, A. Ramsey, M. Zarnstorff, V. Arunasalam, M. Bell, N. Bretz, R. Budny, C. Bush, S. Davis, H. Dylla, P. Efthimion, R. Fonck, E. Fredrickson, H. Furth, R. Goldston, L. Grisham, B. Grek, R. Hawryluk, W. Heidbrink, H. Hendel, K. Hill, H. Hsuan, K. Jaehnig, D. Jassby, F. Jobes, D. Johnson, L. Johnson, R. Kaita, J. Kampershroer, R. Knize, T. Kozub, H. Kugel, B. LeBlanc, F. Levinton, P. LaMarche, D. Manos, D. Mansfield, K. McGuire, D. McNeil, D. Meade, S. Medley, W. Morris, D. Mueller, E. Nieschmidt, D. Owens, H. Park, J. Schivell, G. Schilling, G. Schmidt, S. Scott, S. Sesnic, J. Sinnis, F. Stauffer, B. Stratton, G. Tait, G. Taylor, H. Towner, M. Ulrickson, S. von Goeler, R. Wieland, M. Williams, K.-L. Wong, S. Yoshikawa, K. Young, and S. Zweben, Phys. Rev. Lett. 58, 1004 (1987).

${ }^{25}$ R. J. Fonck, G. Cosby, R. D. Durst, S. F. Paul, N. Bretz, S. Scott, E. Synakowski, and G. Taylor, Phys. Rev. Lett. 70, 3736 (1993).

${ }^{26}$ J. S. Kim, "Experimental measurement of nonlinear energy transfer on the Tokamak Fusion Test Reactor,' Ph.D. thesis, University of Wisconsin, Madison, 1996. 